\author{
Military Technical College \\ Kobry El-Kobbah, \\ Cairo, Egypt.
}

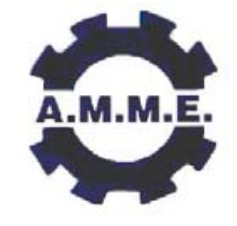

\title{
COMPUTER SIMULATION OF BLANKING PROCESS
}

\author{
AHMED ${ }^{*}$ A. and HASMIRA
}

\begin{abstract}
Blanking process can be simulated on computer and it will aid in die, punch design and press selection. Further effect of punch wear on fracture height and bur height in blank can be ascertained by simulation. This paper demonstrates the blanking process by simulation using ABAQUS v 6.4. For a $2 \mathrm{~mm}$ mild steel sheet the simulation shows that for the punch force of $240 \mathrm{kN}$ the crack starts at $30 \%$ punch penetration depth and punch force suddenly drops to value of $70 \mathrm{kN}$ and remains steady till the blanking is complete. In the elastic plastic range the experimental and simulation results agree well with rising punch force up to the point of creation of crack, however there is slight discrepancy in the down fall of force with depth of penetration of punch. With increase of the thickness of sheet the crack height is reduced and blank quality is improved.
\end{abstract}

\section{KEY WORDS}

Simulation, Blanking, Computer

* Professor, Institute of Manufacturing Engineering, Navy Engineering College, National University of Sciences and Technology, Karachi, Pakistan 


\section{NOMENCLATURE}

D damage variable

$D_{c}$ critical damage value at fracture

$\varepsilon_{\mathrm{pl}}$ plastic part of strain tensor

$\varepsilon_{D}$ threshold logarithmic strain at which Le Maître damage initiates

$\varepsilon_{R}$ logarithmic strain value at fracture

$v$ Poisson's ratio

$\sigma_{\mathrm{H}}$ hydrostatic pressure

$\sigma_{\text {eq }}$ equivalent Von Mises stress

$\sigma_{n}$ stress tensor at increment $(n)$.

$\sigma_{\mathrm{el}}$ initial yield stress

$\tau_{\mathrm{f}}$ friction shear stress

$\mu$ coefficient of friction

\section{INTRODUCTION}

In a blanking process metal is cut by using a punch and die on mechanical or hydraulic press. The quality of sheared edge depends not only on the material but also the process parameters such as the clearance between the punch and the die, the blank holding force, and the sharpness of the edge of punch and die. With the advent of computer the blanking process can be simulated and this can reduce time and cost in engineering decision making and equipment selection. In blanking operation initiation of fracture dominates. The blank is pushed into the die by extrusion like plastic deformation characterized by steadily increasing work force. After a critical deformation, a crack develops and cutting force drops. The aim of paper is to apply FEM along with crack damage model to simulate the blanking process and compare with experimental results. The computer program to simulate the blanking process is ABAQUS/CAE v 6.4. This is finite element program designed to model the behavior of solids and structures under externally applied loading. The blanking process proceeds in five stages [1]. Stage I: As punch pushes into the sheet held by the blank holder, the sheet is elastically deformed. Stage II: As the punch continues to push, first outer fibers of sheet then all inner fibers of sheet between punch and die are subjected to yield strength. Stage III: The sheet fibers between punch and die thin and plastic deformation results in rounding the edge of blank. Here a crack starts developing. Stage IV: A full crack develops and the force pushing the punch is reduced. Stage V: Finally the punch pushes the slug through the die hole and work done due to friction is dissipated. The simulations was considered for blanks of mild steel sheets of thicknesses $2 \mathrm{~mm}, 3 \mathrm{~mm}$, $4 \mathrm{~mm}$, and punch with edge radii of $0.01 \mathrm{~mm}, 0.06 \mathrm{~mm}, 0.12 \mathrm{~mm}, 0.2 \mathrm{~mm}$, die and blank holder edge radii of $0.01 \mathrm{~mm}$. The simulated blanking process is compared with experimental results.

\section{DAMAGE MODELS AND DUCTILE FRACTURE}

As stated earlier in the blanking process, the sheet undergoes a plastic deformation after the elastic deformation and then a crack initiates, the elastic and plastic deformation is taken care by the FEM , however the propagation of crack is simulated by mechanical fracture models which predicts material separation. Three methods are used to realize a fracture in FE-Mesh, that is, element splitting, element separation and 
element deletion. A number of damage models have been developed to try to simulate the development and propagation of ductile fracture. There is no universal model, however, different models are proposed by Husson, Ayada, Oyane, Rice and Tracey, Mclintock, Cockroft / Latham, Lemaitre and Gurson/Needleman and Tvergaard [2,3]. Figure 1 shows the crack initiation and propagation by some models which are quite different to each other [3]

\section{SIMULATION OF BLANKING PROCESS}

\section{Lemaitre Damage Model}

In this paper, Lemaitre model is used for crack initiation and propagation [4]. In Lemaitre model the isotropic damage variable $D$ is the ratio between the total area of the micro cracks and cavities and sectional area of material volume. In incremental form the damage law is:

$\mathrm{dD}=\frac{D_{c}}{\varepsilon_{R}-\varepsilon_{D}}\left(\mathrm{R}_{\mathrm{V}} \mathrm{d} \varepsilon_{\mathrm{pl}}-\varepsilon_{\mathrm{D}}\right)$

where $R_{\mathrm{V}}$ is

$\mathrm{R}_{\mathrm{V}}=\frac{2}{3}(1+v)+3(1-2 v)\left(\sigma_{\mathrm{H} /} \sigma_{\mathrm{eq}}\right)^{2}$

This model requires the knowledge of the three parameters $D_{\mathrm{c}}, \varepsilon_{R}$ and $\varepsilon_{\mathrm{D}}$ which are easily identified by means of a tensile test [5].The initiation of crack starts at any point having damage value of $D_{c}$.

The corresponding strain hardening law takes the non-linear form:

$\sigma=\sigma_{\mathrm{el}}+K \varepsilon_{p l}^{n}$

with the values of $\sigma_{\mathrm{el}}=250 \mathrm{MPa}, K=1048 \mathrm{MPa}$ and $n=0.196$

The rheological constant for the damage law associated with the Le Maitre model are:

$\varepsilon_{D}=0, \varepsilon_{R}=0.8$ and $\mathrm{D}_{\mathrm{c}}=0.37$.

\section{Model Geometry}

Figure 2 shows the geometry of the blanking process components which are punch, die, sheet and the blank holder. The tool wear is simulated by rounding off the cutting edge of punch and die. For blanking a small clearance is kept between punch and die. Following assumptions are made in the analysis:

1.The process is simplified to a two-dimensional situation, under plane-strain conditions, since the punch-die clearance is usually very small compared to the blank diameter.

2.The process is considered quasi-static, and hence the effects of strain rate are neglected. 
3.The sheet material is considered as elastoplastic while the punch and die are defined as rigid

The friction contact between sheet and tool follows Coulomb's law:

$\tau_{\mathrm{f}}=\mu \sigma_{n}$

\section{Issues In Modeling By ABAQUS}

There are a number of issues that should be considered when modeling contact problems in ABAQUS/Standard that involve rigid surfaces. Some of important issues are $[6,7]$ :

- ABAQUS manual refers to use of ABAQUS/Standard and ABAQUS/ Explicit. ABAQUS/Standard solves a system of equations implicitly at each solution increment. In contrast, ABAQUS/ Explicit makes a solution forward through time in small time increments without solving a coupled system of equations at each increment

- The rigid surface is always the master surface in contact interaction.

- The rigid surface should be large enough to ensure that slave nodes do not slide off and 'fall behind' the surface. If this happens, the solution usually will fail to converge. Extending the rigid surface of including corners along the perimeter will prevent slave nodes from falling behind the master surface.

- The deformable mesh must be refined enough to interact with any feature on the rigid surface. There is no point in having a $10 \mathrm{~mm}$ wide feature on the rigid surface if the deformable elements that will contact it are $20 \mathrm{~mm}$ across. The rigid feature will just penetrate into the deformable surface. With sufficiently refined mesh on the deformable surface. ABAQUS/Standard will prevent the rigid surface from penetrating the slave surface.

- The contact algorithm in ABAQUS/Standard requires the master surface of a contact interaction to be smooth. Rigid surfaces are always the master surface and so should always be smoothed. ABAQUS/Standard does not smooth discrete rigid surfaces. The level of refinement controls the smoothness of a discrete rigid surface. Analytical rigid surfaces can be smoothed by defining a fillet radius that is used to smooth any sharp corners in the rigid surface.

\section{Input Data For Modeling}

1. Simulation of mild steel sheets with thickness of $2 \mathrm{~mm}, 3 \mathrm{~mm}$ and $4 \mathrm{~mm}$ called the blank, and the tools that are the punch with edge radius $R_{p}=0.01 \mathrm{~mm}$, die and blank holder with edge radius $R_{d}=0.01 \mathrm{~mm}$ that contact the blank.

2. Simulation of mild steel sheets with thickness $3 \mathrm{~mm}$ called the blank, and the tools that are the punch with different edge radii $R_{p}=0.06 \mathrm{~mm}, 0.12 \mathrm{~mm}$ and $0.2 \mathrm{~mm}$, die and blank holder with edge radius $R_{d}=0.01 \mathrm{~mm}$ that contact the blank.

The tools can be modeled as rigid surfaces because they are much stiffer than the blank. A two-dimensional, plane strain model is used. The assumption that there is no strain in the out-of-plane direction of the model is valid if the structure is long in this direction. Only half of the blanking is modeled because the blanking process is symmetric about a plane along the center of the blank. 
The blank is made from mild steel having $E=207.0 \times 10^{9} \mathrm{~Pa}, \mathrm{v}=0.29$. An inelastic stress-strain behavior for mild steel is shown in Table1. The material undergoes considerable work hardening as it deforms plastically. It is likely that plastic strains will be large in this analysis. Therefore, hardening data are provided up to $50 \%$ plastic strain.

\section{Part Definition}

ABAQUS /CAE is started by entering the part module. Four parts are created. They are: one deformable part representing the blank and other three are rigid parts representing the tools. The deformable part blank is named Blank. For rigid tools the parts created are Punch, Holder and Die. The edge radius of the punch, $R_{p}$ is created with required values. A rigid body reference point must be created. The point at the center of the arc in the view port is selected as the rigid body reference point. Next, two additional analytical rigid parts named Holder and Die are created using the same process as creating the punch, with the edge radius of $0.01 \mathrm{~mm}$ representing the blank holder and rigid die, respectively.

In the property module, a material named Steel $2 \mathrm{~mm}$ with properties given in Table 1 is created. A homogeneous solid section named BlankSection is also created that refers to material Steel. The blank is selected from the list of available parts in the Part list located under the toolbar, and the section is assigned to the blank. The blank is going to undergo significant rotation as it deforms. Reporting the values of stress and strain in a coordinate system that rotates with the blank's motion will make it much easier to interpret the results. Therefore, a local material coordinates system that is aligned initially with the global coordinate system but moves with the elements as they should be created. To do this, a rectangular datum coordinate system is created using the Create Datum CSYS: 3 Points tool. From the main menu, select Assign $\rightarrow$ Material Orientation. The blank is selected as the region to which the local material orientation will be assigned, and the datum coordinate system in the view port is picked as the CSYS (select Axis-1 and accept 0.0 for the additional rotation options).

\section{Assembling the Parts}

The assembly module is entered by instancing the blank. Then, the rigid tools are instanced and positioned. The clearance between punch and die is set as $1 \%$ of the thickness of plate.

\section{Geometry Sets}

At this point it is convenient to create the geometry sets that will be used to specify loads and boundary conditions and to restrict data output. Six sets are: one at each body reference point, one at symmetry plane of the blank, and one at each end of the blank midplane. The last two sets require that a vertex first exist at these locations, that is, an edge partition can be performed to satisfy this requirement.

Begin by creating the following geometry sets:

- RefPunch at the punch rigid body reference point.

- RefHolder at the holder rigid body reference point.

- RefDie at the die rigid body reference point. 
- $\quad$ Center at the left vertical edge (symmetry plane) of the blank.

Next, partition the vertical edges of the blank in half and create sets at the new vertices by partitioning the left and right vertical edges of the blank using the Partition Edge: Enter Parameter tool. For each edge, specify a normalized edge parameter of 0.5 to partition the edge in half. Each vertical edge of the blank now has a vertex located on the blank midplane Define a set at the left midplane vertex named MidLeft and one at the right midplane vertex named MidRight.

\section{Defining the contact interactions}

Contact must be defined between the top of the blank and the punch, the top of the blank and the blank holder, and the bottom of the blank and the die. The rigid surface must be the master surface in each of these contact interactions. Each contact interaction must refer to a contact interaction property that governs the interaction behavior. In this analysis, the friction coefficient between the blank and the punch is assumed as zero. The friction coefficient between the blank and the other two tools is assumed to be 0.1 . Therefore, two contact interaction properties must be defined that are one with friction and one without it. In the Interaction module, the following surfaces are defined.

- BlankTop on the top edge of the blank

- BlankBot on the bottom edge of the blank

- DieSurf on the side of the die that faces the blank

- HolderSurf on the side of the holder that faces the blank

- PunchSurf on the side of the punch that faces the blank

Two contact interaction properties are defined. The first one is named as NoFric. Since frictionless contact is the default in ABAQUS, the default property settings is accepted for the tangential behavior. The second property is named as Fric. For this property, Penalty friction formulation with a friction coefficient of 0.1 is used. Finally, the interactions between the surfaces are defined by referring to the appropriate contact interaction property for each definition. In all cases, the interactions are defined in the Initial step and the default finite-sliding formulation (Surface-to-surface contact (Standard)) is used. The following interactions are defined:

- Die-Blank between surfaces DieSurf (master) and BlankBot (slave) referring to the Fric contact interaction property.

- Holder-Blank between surfaces HolderSurf (master) and BlankTop (slave) referring to the Fric.

- Punch-Blank between surfaces PunchSurf (master) and BlankTop (slave) referring to the NoFric contact interaction property.

\section{Defining Step and Output Requests.}

The simulation consists of five steps. Since the simulation involves material, geometry, and boundary nonlinearities, general steps are used. A brief summary of each step is given hereafter.

\section{Step1- Push the blank holder and the die together to hold the blank}

This step is intended to establish firm contact between the blank and the blank holder. In this step the endpoints of the midplane of the blank are fixed in the vertical direction 
to prevent the blank from moving initially, and the blank holder is pushed down onto the blank using a displacement boundary condition. The step is to be completed in one increment. In addition the die is moved upward to establish a firm contact between die and blank. Care is to be taken to apply displacement which does not cause plastic yielding. The blank holder and die are constrained in degrees of freedom 1 and 6 : Degree of freedom 1 is motion in horizontal direction and degree of freedom 6 is the rotation in the plane of the model. All the boundary conditions for the rigid surfaces are applied to their respective rigid body reference nodes. The punch is constrained completely, and symmetric boundary constraints are applied on the region of the blank lying on the symmetry plane (geometry set Center). Table 2 summarizes the boundary conditions applied in this step:

\section{Step 2 - Remove the middle constraint at right}

Since contact was established between the blank and the blank holder and die in the previous step, the constraint on the right end of the blank midplane is no longer necessary and is removed in a second static, general step. This step should also be completed in a single increment because the only change being made to the model is the removal of the vertical constraints on the blank.

\section{Step 3 - Apply prescribed force on blank holder}

The magnitude of the blank holder force is a controlling factor in many blanking processes. Therefore, it needs to be introduced as a variable load in the analysis. In this step the boundary condition used to move the blank holder down will be replaced with a force. In this simulation the required blank holder force is $440 \mathrm{kN}$. Generally, care should be taken to ensure that the newly applied force is of the same order as the reaction force generated from the boundary condition so that the contact condition does not change significantly.

\section{Step 4 - Move the punch down a little while applying a small pressure to blank top}

At the beginning of the analysis the punch and the blank are separated to avoid any interference while contact is established between the blank and the die and blank holder. In this step the punch is moved down in 2-direction ( vertical motion) just enough to achieve contact with the blank. In addition, the vertical constraint on the left end of the blank midplane is removed and a small pressure is applied to the top surface of the blank to pull it onto the surface of the punch. In this analysis, a pressure magnitude $(1000 \mathrm{~Pa})$ that produced a force on the blank is used that is three orders of magnitude lower than the blank holder force. A positive pressure acts on the surface. However, in this simulation a negative pressure is used so that the pressure act out of the surface. This technique is used to prevent chattering of the BlankTop and Punch surfaces.

\section{Step 5 - Move the punch with full extent}

In the fifth final step the pressure load applied to the surface BlankTop will be removed and the punch will be moved down to complete the blanking operation. The reaction force at the punch reference point is requested to be written every increment as history data.

\section{Monitoring the value of a degree of freedom}

The value of a degree of freedom can be requested at one selected point. The value of the degree of freedom is shown in the Job Monitor and is written at every increment to 
the status file and at specific increments during the course of an analysis to the message file. In addition, a plot of the degree of freedom value over time appears in a new viewport monitor the progress of the solution. In this model, the vertical displacement (degree of freedom 2) of the punch's reference node can be monitored throughout each step. Before proceeding, the first analysis step (Holder force) is activated by selecting it from the Step list located under the toolbar. The monitor definition applied for this step is propagated automatically to all the subsequent steps.

\section{Mesh creation and job definition}

Either incompatible mode or reduced-integration elements are suitable for this analysis. In this analysis, reduced-integration elements with enhanced hourglass control are used. Reduce-integration elements help decrease the analysis time, and enhanced hourglass control reduces the possibility of hourglassing in the model. In the Mesh module, the blank is meshed using CPE4R elements with enhanced hourglass control. The edges of blank are seed by specifying the number of elements along each edge. 40 elements along the horizontal edges of the blank and 16 elements along each region on the vertical edges of the blank are specified. The total numbers of nodes and elements were 1143 and 1006 respectively. Finally, in the Job module, the analysis is submitted and monitored.

\section{RESULTS AND DISCUSSION}

Figure 3 shows the simulation of punch force vs punch penetration for a $2 \mathrm{~mm}$ mild steel sheet . The force rises initially up to about $240 \mathrm{kN}$ with elastic and plastic yielding. The crack starts and force drops to $70 \mathrm{kN}$. The crack propagates now at nearly constant force. Figure 4 shows the simulation of punch force vs punch penetration for mild steel sheet of $4 \mathrm{~mm}$. The curve is slightly different from $2 \mathrm{~mm}$ mild steel sheet because of the effect of increased thickness of sheet. There seems to be a resistance in the initiation of crack as the crack starts at about $70 \%$ punch penetration. Further the peak force lowers to about $140 \mathrm{kN}$ with penetration up to $80 \%$. Unlike $2 \mathrm{~mm}$ sheet the crack does no propagate at a nearly constant force but propagates with the reduction of force. Further the fracture height is less, that is, fracture height reduces as the sheet thickens.

Figure 5 shows comparison of simulation results with that of the experiment. The experimental results are taken from the research paper by Ridha Hambli[5].The experimental and simulation results are for $3 \mathrm{~mm}$ mild steel sheet with punch edge radius of punch $0.01 \mathrm{~mm}$. The Figure shows good agreement in experimental and simulation results in elastic and plastic range but with some deviation in force when the crack develops. However, the final force in simulation required for separation of blank from sheet tallies with that of experiments. The deviation in the stage of development of crack is expected because of the damage model as no damage model depicts the true crack initiation phenomenon.

Figure 6 shows the experimental results [5] for punch force vs punch penetration for 3 $\mathrm{mm}$ thick mild steel sheet for different punch edge radii. The increase of punch edge radius exhibits the wear of punch. Figure 7 shows the simulation results for the similar data as of Figure 6 . The punch forces in elastic plastic region are in close agreement with those from experiment that is all reach $250 \mathrm{kN}$ and also have same start of crack at punch penetration of $30 \%$ depth. However the final force requirement for separation of blank from sheet is different in case of experimental results - a lower force of $50 \mathrm{kN}$ for 
the worn punch of edge radius of $0.2 \mathrm{~mm}$.In case of simulation the force of separation of blank from sheet is nearly same for fine tool and worn tool again because of the limitation of damage model. However, by simulation one can estimate the punch force requirement to determine the capacity of press needed. Also the effect of punch wear on fracture height and bur height can be determined which in turn signify the quality of the blank.

\section{CONCLUSION}

The ABAQUS v 6.4 satisfactorily simulates the blanking process. In the elastic plastic range simulation is well in agreement with experimental results. After plastic deformation a crack develops and the FEM model is now incorporated with a crack damage model. Lemaitre damage model approximates to crack propagation. As the crack develops the punch force is reduced considerably and punch penetrates depending on the punch edge radius. Higher the punch edge radius more is the punch penetration. With increased thickness of sheet the crack height is reduced and blank quality is improved. The punch wear has no marked effect on the force required in elastic plastic region, however, in the fracture zone there is marked difference as the characteristic of fracture propagation and resistance to fracture with respect to punch force changes as observed in experiments [5]. However this marked difference is not much obvious in simulation because of the use of damage model.

\section{ACKNOWLEDGEMENT}

The research was undertaken at School of Mechanical Engineering, Universiti Sains Malaysia.

\section{REFERENCES}

[1] Klingenberg,W and Singh,U.P." Comparison of Two Analytical Methods of Blanking and Proposal of a New Model"Int. J. Machine Tools and Manufacture.,45pp 519-527(2005)

[2] Klingenberg,W and de Boer, T.W."Condition Based Maintenance in Punching/Blanking of Sheet Metal", Int. J. Machine Tools and Manufacture., In press.

[3] Klocke,F.,Sweeny,K, and Raedt,H.W.'Improved Tool Design for Fine Blanking through the Application of Numerical Modeling Techniques",J.of Material Processing Technology 115 pp70-75 (2001).

[4] J.Lemaitre,"A Continuous Damage Mechanics Model for Ductile Fracture", J.of Engineering Materials and Technology-TransanctionsASME 107pp 83-89 (1985).

[5] Hambli, R and Potiron,A.," Finite Element Modeling of Sheet Metal Blanking Operations with Experimental Verification", J. of Material Processing Technology, Vol.102, Issues 1-3, pp 257-265 (May 2000).

[6] ABAQUS Inc.Getting Started with ABAQUS Version 6.4 (2003)

[7] ABAQUS Inc ABAQUS/CAE User's Manual Version 6.4 (2003) 
Table 1. Yield stress-plastic strain data for mild steel.

\begin{tabular}{|c|c|}
\hline Yield stress $(\mathrm{Pa})$ & Plastic strain \\
\hline $400.0 \mathrm{E} 6$ & 0.0 \\
\hline $420.0 \mathrm{E} 6$ & $2.0 \mathrm{E}-2$ \\
\hline $500.0 \mathrm{E} 6$ & $20.0 \mathrm{E}-2$ \\
\hline $600.0 \mathrm{E} 6$ & $50.0 \mathrm{E}-2$ \\
\hline
\end{tabular}

Table 2. Summary of boundary conditions applied in Step 1.

\begin{tabular}{|c|c|c|}
\hline BC Name & Geometry Set & BCs \\
\hline CenterBC & Center & XSYMM \\
\hline RefDieBC & RefDie & $\mathrm{U} 1=$ UR3 $=0.0$, U2 $=1$. E-08 \\
\hline RefHolderBC & RefHolder & $\mathrm{U} 1=\mathrm{UR3}=0.0, \mathrm{U} 2=-1 . \mathrm{E}-08$ \\
\hline RefPunchBC & RefPunch & $\mathrm{U} 1=\mathrm{U} 2=\mathrm{UR} 3=0.0$ \\
\hline
\end{tabular}

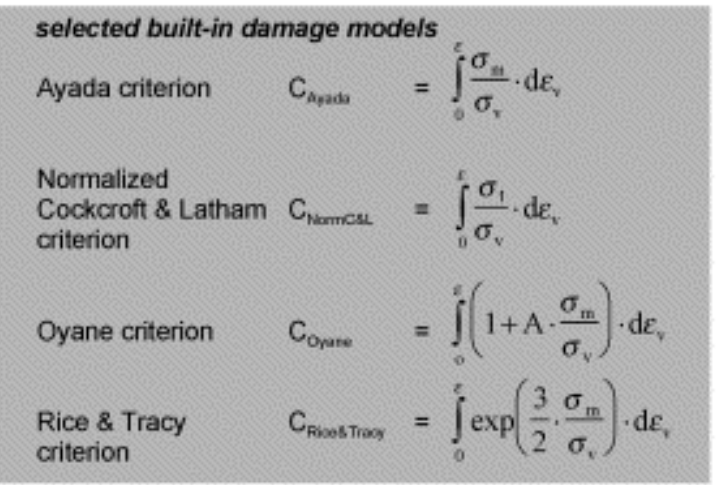

Material : C16

Thickness : $7.7 \mathrm{~mm}$
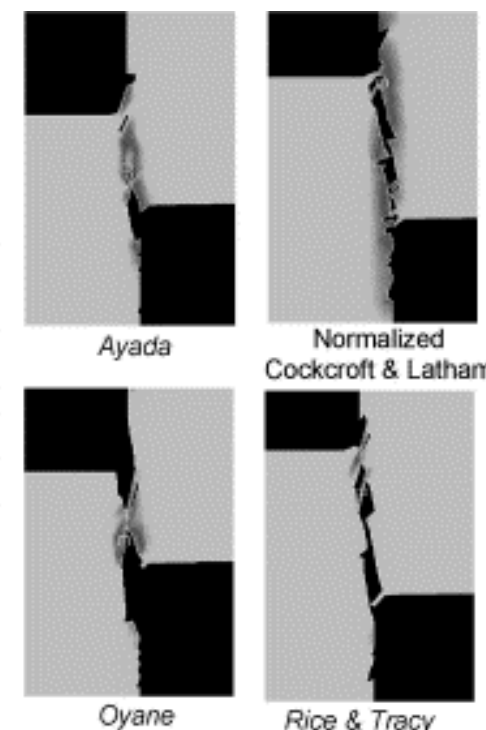

Normalized Cockcroft \& Latham

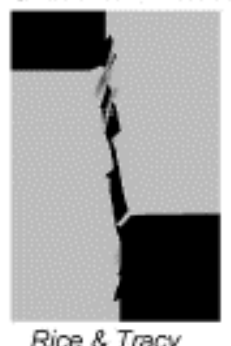

Rice \& Tracy

Figure 1 Selected damage models for crack prediction by Klocke,et.al 


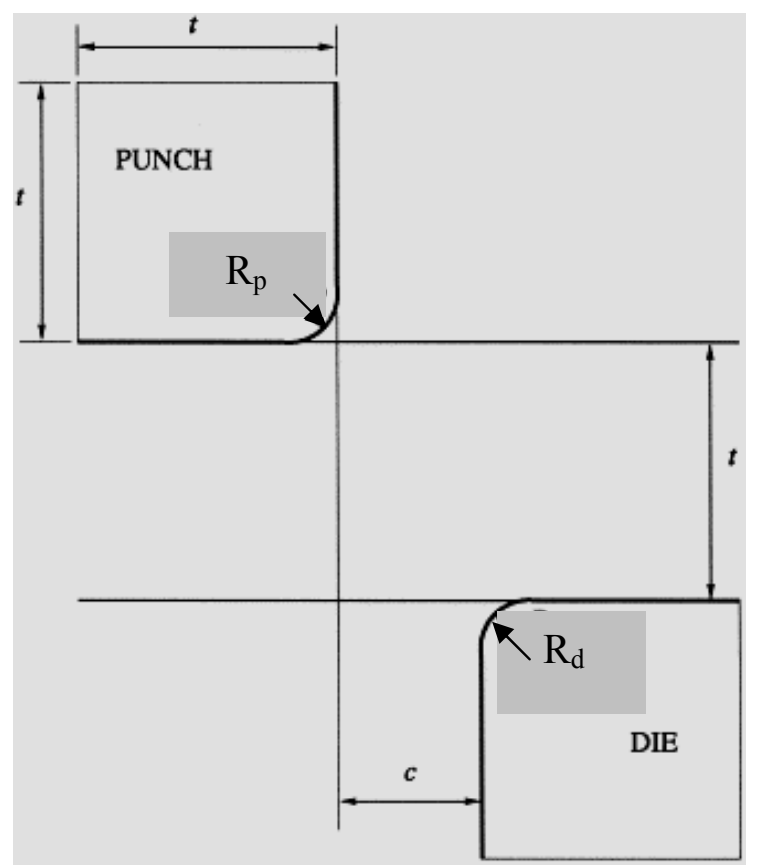

Figure 2. Geometry of punch, die and sheet and clearance

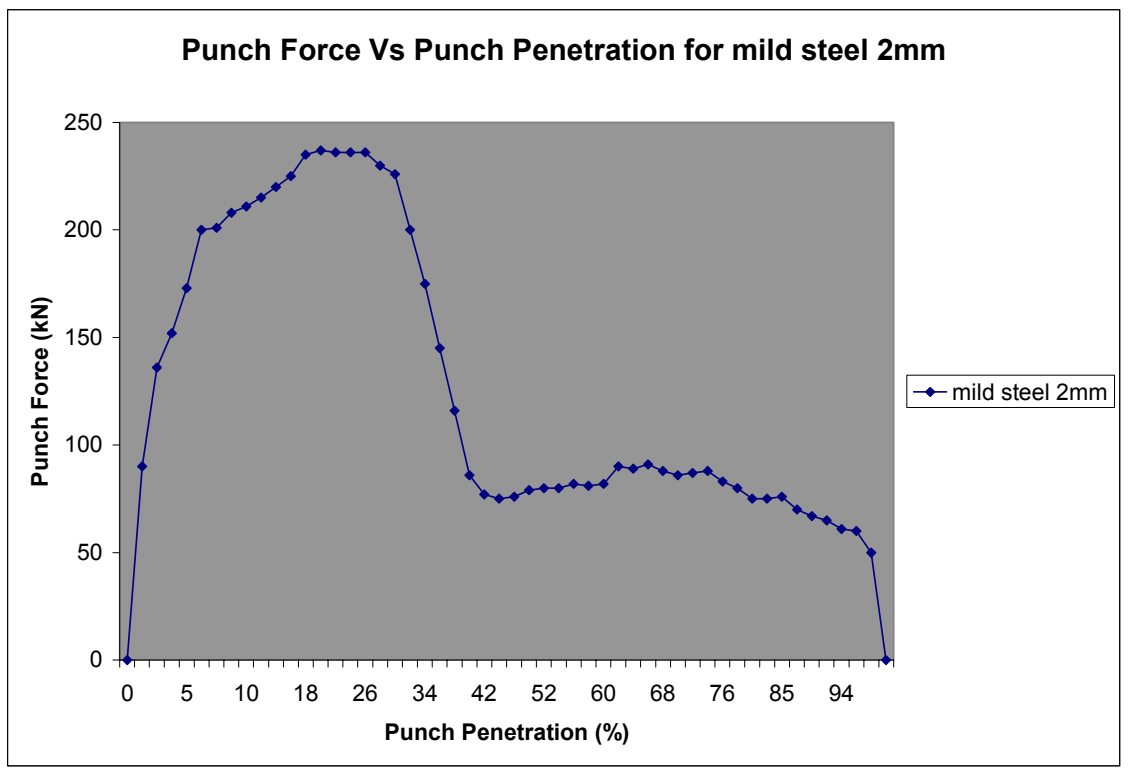

Figure 3 Punch force vs punch penetration of blanking process ( $2 \mathrm{~mm} \mathrm{MS} \mathrm{Sheet)}$ 


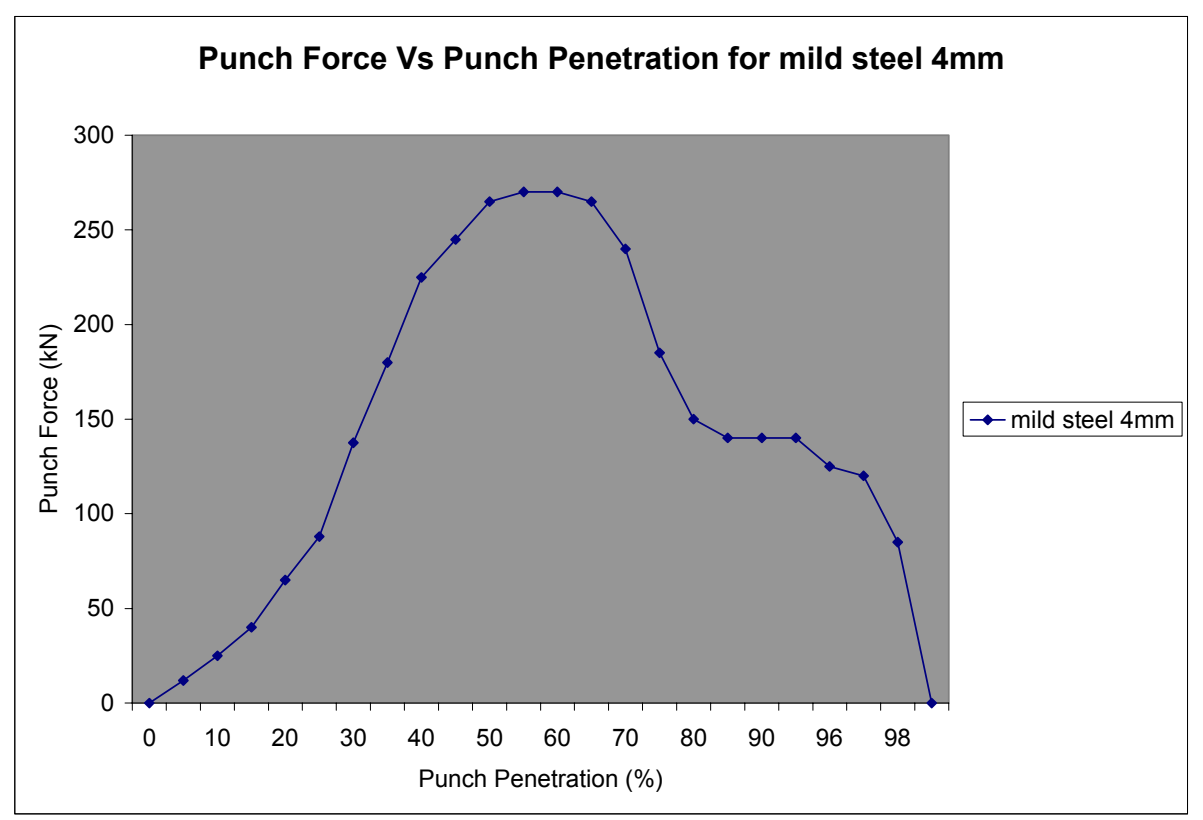

Figure 4 Punch force vs punch penetration of blanking process ( $4 \mathrm{~mm} \mathrm{MS} \mathrm{Sheet)}$

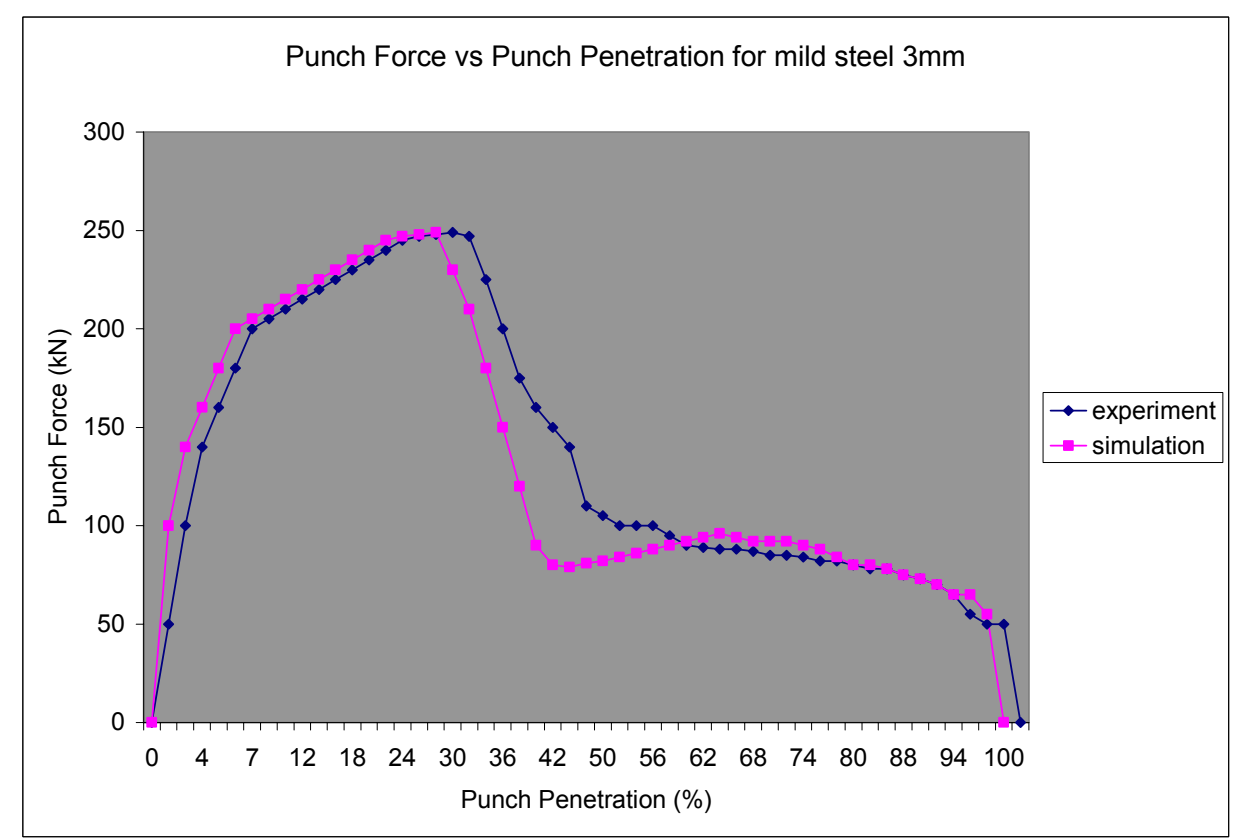

Figure 5 Simulation compared with experimental results [5] for 3mm MS Sheet 


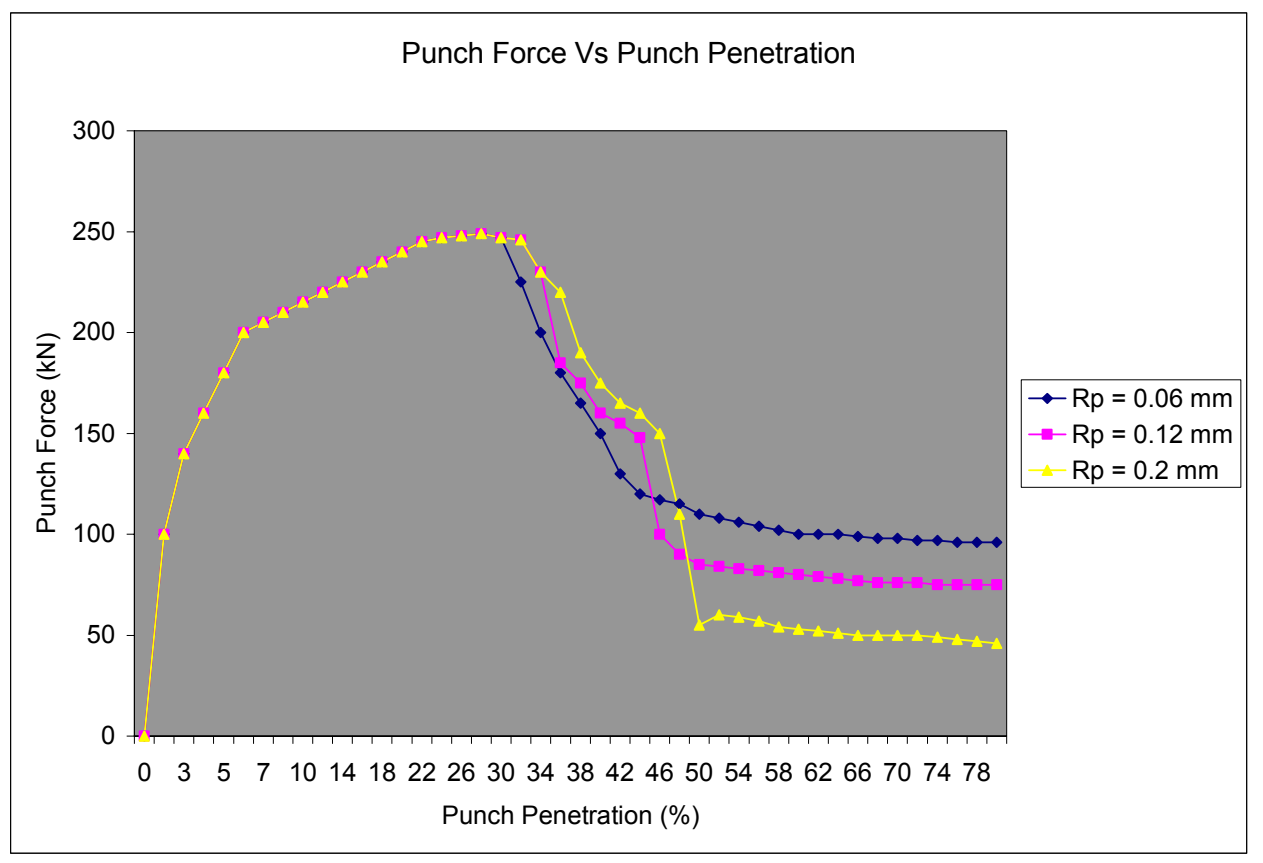

Figure 6 Experimental results for $3 \mathrm{~mm}$ MS Sheet for various punch edge radii[5]

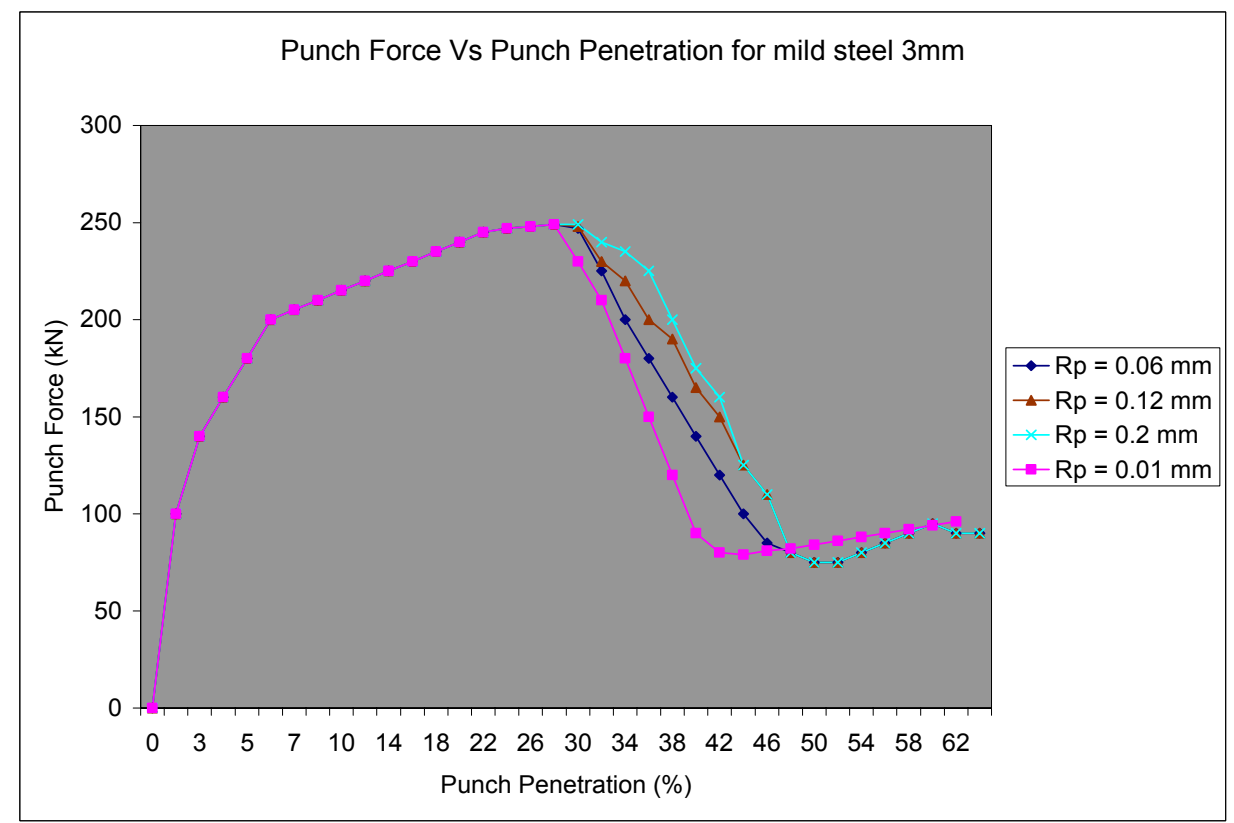

Figure 7 Simulation results for various punch edge radius for blanking of $3 \mathrm{~mm} \mathrm{MS}$ Sheet. 